Recepción: 03/10/2018

Evaluación: 03/ 12/2018

Aprobación: 06/05/2019

Artículo de Investigación- Científica

DOI: https://doi.org/10.22267/rhec.192222.52

\title{
Propuesta de formación del maestro-mediador para la consolidación de la cultura de paz en Colombia ${ }^{1}$
}

\author{
Karol Restrepo Mesa ${ }^{2}$ \\ Universidad Pontificia Bolivariana, Colombia
}

\section{Resumen}

Este artículo de investigación analiza la relación entre Educación para la Paz (EpP), Pedagogía de la Memoria y Comunicación, como elementos de la formación del maestro, que facilitan la consolidación de una cultura de paz en el marco del posacuerdo, en Colombia. Adicionalmente, analiza la injerencia de los modelos pedagógicos a la luz de los procesos de construcción de paz, en el contexto colombiano. Y, por último, sugiere una propuesta temática a los programas de formación de maestros, en la búsqueda de formar sujetos que hicieran del acto educativo un proceso lleno de sentido, para que garantizaran a sus estudiantes aprendizajes significativos desde la experiencia. Así, el maestro se convertirá en estratega, al tomar el rol de mediador para construir puentes de intersubjetividad que permitieran el diálogo y la convivencia, proceso que servirá de punto de partida para reflexiones que desemboquen en modelos de formación continuada de maestros y políticas educativas futuras, con miras a alcanzar una paz sostenible y un país más equitativo.

Palabras clave: Comunicación; Cultura de Paz; Educación para la Paz; Formación de Docentes.

$1 \quad$ El artículo surge en el marco del proyecto Creando Paz: recursos culturales en experiencias de mediación y gestión constructiva de conflictos y su aporte a la formación de competencias ciudadanas y construcción de cultura de paz, ejecutado por la Universidad Pontificia Bolivariana y coejecutado por Corporación Región, desde el año 2017; financiado por Colciencias a través del Programa Nacional de Ciencia, Tecnología e Innovación en Ciencias Humanas Sociales y Educación (Código Colciencias N 121074054774, Radicado interno CIDI-UPB: 745B-03/17-16).

2 Comunicadora Social, Estudiante de la Maestría en Educación. Grupo de investigación: Pedagogía y Didácticas de los Saberes de la Universidad Pontificia Bolivariana, Colombia. Correo electrónico: karol.restrepo@upb.edu.co. Código ORCID: https://orcid.org/0000-0001-9208-9468 


\title{
Proposal of a teacher-facilitator training program within the peace culture in Colombia
}

\begin{abstract}
This research article analyzes the relationship between Education for Peace (EpP), Pedagogy of Memory and Communication, as elements of teacher training, that facilitate the consolidation of a peace culture within the context of the post-conflict in Colombia. In addition, it analyzes the interference of pedagogical models in the light of peacebuilding processes in the Colombian background. And finally, it suggests a thematic proposal to teacher training programs in search of training subjects that make the educational act a meaningful process, so that they guarantee their students with significant learning from experience. In this way, the teacher will become a strategist, taking on the role of facilitator to build bridges of intersubjectivity that allow dialogue and coexistence; a process that will serve as a starting point for certain considerations that will lead to models of continuous teacher training and future educational policies, to achieve a sustainable peace and a more equitable country.
\end{abstract}

Keywords: Communication; Culture of Peace; Education for Peace; Teacher Training. 


\section{Proposta de capacitação do professor-mediador para a consolidação da cultura de paz na Colômbia}

\section{Resumo}

Este artigo de pesquisa analisa a relação entre Educação para a Paz (PPE), Pedagogia da Memória e Comunicação, como elementos da formação de professores que facilitam a consolidação de uma cultura de paz no âmbito do pós-acordo na Colômbia. Além disso, analisa a interferência de modelos pedagógicos à luz dos processos de construção da paz no contexto colombiano. E, finalmente, sugere uma proposta temática para os programas de formação de professores, na procura de formar sujeitos que tornem o ato educacional um processo significativo, para que garantam aos alunos um aprendizado significativo da experiência. Assim, o professor se tornará um estrategista, assumindo o papel de mediador na construção de pontes de intersubjetividade que permitam o diálogo e a convivência; Esse processo servirá como ponto de partida para reflexões que levam a modelos de formação continuada de professores e futuras políticas educacionais, com vistas a alcançar a paz sustentável e um país mais equitativo.

Palavras-chave: Comunicação; Cultura de paz; Educação para a Paz; formação de profesores. 


\section{Introducción}

En el contexto del posacuerdo, en Colombia, la Educación para la Paz $(\mathrm{EpP})$ es el proceso privilegiado de enseñanza, aprendizaje y des-aprendizaje para el fortalecimiento de la cultura de paz a través de la formación en libertad y autonomía. Esta estrategia se mediatizó en el país a través de la Pedagogía de la Memoria, entendida como una reflexión ética sobre la realización de ejercicios mnemotécnicos en colectivo, para reconocer en el pasado factores que estuvieran en relación con lo que sucede en el presente ${ }^{3}$ y establecer, así, un nuevo comienzo.

En este sentido, este artículo estructura las articulaciones posibles entre EpP, Pedagogía de la Memoria y Comunicación, como elementos para una propuesta de formación del Maestro- Mediador que consolida una cultura de paz y que cumple con el compromiso social de desarrollar un país más equitativo. En esta coyuntura, una tarea para el maestro es disminuir la violencia en sus relaciones a través de la construcción de un ámbito en el que cada persona pueda expresarse y avanzar en las relaciones, la comunicación y el inter-aprendizaje ${ }^{4}$ desde el lenguaje, el encuentro y la experiencia. ${ }^{5}$

Esta propuesta sugiere un contenido diferenciador que responde a las necesidades del contexto, en el marco del proyecto Creando Paz. Y su desarrollo responde a la pregunta sobre: ¿Cómo se articulan los conceptos Educación para la Paz, Pedagogía de la Memoria y Comunicación, como elementos de la formación del maestro, que le faciliten consolidar una cultura de paz, en el marco del posacuerdo en Colombia? Para responder a este cuestionamiento, se definió como objetivo general establecer las articulaciones posibles entre estos conceptos; y como objetivos específicos se definieron, en primer lugar, interpretar el tipo de articulación y, seguidamente, generar una propuesta temática para la formación del maestro-mediador.

3 Fernando Bárcena Orbe, "Pedagogía de la Memoria y transmisión del mundo. Notas para una reflexión", Con-ciencia social. No. 15 (2011): 109-118.

4 Daniel Prieto, La comunicación en la educación (Buenos Aires: Ediciones Ciccus La Crujía, 1999).

5 Jorge Larrosa, Entre las lenguas: lenguaje y educación después de Babel (Barcelona: Laertes, 2003). 
La metodología utilizada, en un primer momento, para el desarrollo de este trabajo, fue la revisión documental desde el enfoque hermenéutico. Por una parte, se abordaron los enfoques teóricos de Educación para la Paz ofrecidos por María Zambrano, Vicenç Fisas, Xesus Jares y Federico Mayor Zaragoza; por la otra, se analizaron las conceptualizaciones de Pedagogía de la Memoria generadas por Graciela Rubio y Susana Sacavino y, por último, se retomaron los puntos de vista de Jesús Martín-Barbero, Daniel Prieto y Óscar Saldarriaga, para establecer la insoslayable relación entre Comunicación-Educación. En la segunda parte de la investigación, se realizó un Estudio de Caso Etnográfico en el cual se recogió información por medio de entrevistas individuales semiestructuradas a profundidad y observación en campo. Por último, los datos recolectados se analizaron por medio de triangulación, codificación y análisis de contenidos y materiales.

El desarrollo de este artículo se presenta así: primero, se aborda la descripción de la metodología y el problema de investigación; luego, se elabora el concepto de Educación para la Paz, como estrategia para la consolidación del posacuerdo en Colombia; a posteriori, se presenta el concepto de Pedagogía de la Memoria, su origen y conceptualización, y se finaliza con la sugerencia de perfil del Maestro-mediador y la propuesta temática para su formación como un constructor de paz desde las aulas.

La investigación se asumió desde el paradigma cualitativo, mediante el cual mujeres víctimas y sobrevivientes del conflicto social armado, en el municipio de Granada-Antioquia, presentaron formas posibles de reconstruir el tejido social; entre estas posibilidades mencionan al sistema educativo y a los maestros como engranaje clave del proceso. Strauss y Corbin señalan que la investigación cualitativa: "Puede tratarse de investigaciones sobre la vida de la gente, las experiencias vividas, los comportamientos, emociones y sentimientos, así como al funcionamiento organizacional, los movimientos sociales, los fenómenos culturales y la interacción entre las naciones". ${ }^{6}$

Según ellos, existen tres componentes principales de este paradigma, que se vieron reflejados en esta investigación: 1) los datos, 2) los procedimientos utilizados para interpretarlos y organizarlos, como la codificación, y 3 ) los informes

$6 \quad$ Anselm Strauss y Juliet Corbin, Bases de la investigación cualitativa. Técnicas y procedimientos para desarrollar la teoría fundamentada (Medellín: Universidad de Antioquia, 2002), 20. 
escritos y verbales, sobre los resultados de la investigación; se enfatizó más en los aspectos epistemológicos, que guiaron el diseño de toda la investigación.

El enfoque cualitativo favoreció el alcance de los objetivos de esta investigación, en la medida en que generó las oportunidades necesarias para que las voces de los sujetos que conformaron la experiencia objeto de estudio se escucharan, analizaran e interpretaran, para otorgarle el respectivo valor a la subjetividad de sus relatos y sus testimonios. Así, esta metodología, pertinente, facilitó comprender el significado de la experiencia objeto de estudio, a la par que proporcionó detalles complejos de los fenómenos analizados, difíciles de extraer o de aprehender por métodos de investigación más convencionales.

Este proceso se abordó desde el método del Estudio de Caso Etnográfico, sugerido por Helen Simons, ${ }^{7}$ que permitió utilizar diversas técnicas para la obtención de la información y el análisis de los datos recogidos, como la realización de 14 entrevistas individuales semiestructuradas a profundidad, que se registraron en audio durante las visitas al campo; además, las observaciones, percepciones y reflexiones se consignaron en un diario de campo. Adicionalmente, se reunió información a través de documentos (actas), grabaciones, fotografías y otros objetos, que permitieron identificar las apreciaciones personales, el sentir y el grado de afectación que ha tenido la experiencia en las formas de reparación que se han emprendido y las formas en que se constituyen en recursos formativos.

A esto se suman las técnicas de análisis de los datos recolectados, como la triangulación, la categorización y el análisis de contenido y materiales. El análisis de contenidos se convierte en la técnica fundamental para comprender el significado de las situaciones de tensión alrededor del objeto de estudio, a través de la lectura sistemática, el uso de rejillas para rotular y la búsqueda de regularidades y rarezas.

En suma, se buscó que todos los integrantes del programa, desde su rol, participaran del proceso y proporcionaran información a partir de su experiencia y mirada particular. Esto permitió la obtención de información amplia y profunda y garantizó, en buena medida, procesos de confiabilidad y validez en los resultados de la investigación.

Helen Simons, El Estudio de Caso: Teoría y práctica (Madrid: Morata, 2011). 
La problemática de esta investigación se puede presentar sintéticamente de la siguiente forma: en un país como Colombia, con un conflicto social armado con más de seis décadas de antigüedad, los maestros se convierten en el puente fundamental para la reconstrucción de tejido social desde la Escuela, al establecer imaginarios colectivos de paz que permitan a la Educación para la Paz, desde la Pedagogía de la Memoria, constituir mediaciones y nuevas formas de convivir entre todos los actores de una comunidad educativa.

Durante el conflicto armado en el país, los actos violentos pueden invadir múltiples realidades, pero los maestros-mediadores elaboran escenarios de paz en diferentes espacios de intervención. Esto solo es posible si los maestros, desde su proceso de pregrado, formación continua y posgrado, conocen el pasado del país para intervenir adecuadamente en el presente, con el uso de las mejores estrategias pedagógicas y modificar el futuro hacia la paz sostenible.

Existen procesos investigativos que evidencian las articulaciones que se pueden establecer para formar un maestro pertinente, que forjase una cultura de paz, mediante el uso de la Pedagogía de la Memoria y las estrategias de comunicación. Pese a esto, no pareciera que las propuestas gubernamentales para la construcción de una cultura de paz reconocieran de manera clara este rol fundamental de los maestros como mediadores.

Y este es el vacío que pretende llenar este trabajo de investigación: demostrar que el maestro-mediador es el agente fundamental de la comunidad educativa, para consolidar una cultura de paz sostenible desde la mediación del conocimiento, por medio de la reflexión crítica sobre lo sucedido en el pasado y el uso de la comunicación asertiva con la comunidad educativa, especialmente con los estudiantes.

\section{Educación para la Paz: estrategia para la consolidación del posacuerdo}

En el contexto del posacuerdo, en Colombia, la Educación para la Paz (EpP) es un encuentro de subjetividades que aprovecha la riqueza de conocimiento, valores, actitudes y aptitudes de cada individuo en el camino de enseñanza, aprendizaje y des-aprendizaje para el fortalecimiento de la cultura de paz, por medio de la formación en libertad y autonomía, que genera subprocesos de mediación y gestión constructiva de conflictos para movilizar a la sociedad hacia el cambio, con base en valores como: justicia, verdad, confianza y seguridad. 
El objetivo principal de la EpP es sensibilizar y generar conciencia en torno a las ideas sobre la paz positiva y la perspectiva creativa del conflicto, ${ }^{8}$ al desarrollar autonomía y confianza en cada uno de los actores implicados en este proceso. La Educación para la Paz se caracteriza primordialmente porque busca la democratización de las estructuras sociales, entre ellas la del sistema educativo; además, apela por la coherencia entre los fines y los medios empleados para educar ${ }^{9}$ y propicia el diálogo constante entre los valores, principios, símbolos y signos que constituyen la cultura de paz.

Los contenidos de la EpP, además de enfocarse especialmente en este tipo de cultura, se orientan igualmente sobre los conflictos y la forma de tramitarlos, la educación para la disidencia, la indignación, la desobediencia responsable, la elección con conocimiento y la reflexión para formar personas con criterio y evitar que se repitieran en el sistema las estructuras que coartan la libertad e inhiben la paz. ${ }^{10}$

La Educación para la Paz, en Colombia, se retomó como una estrategia para consolidar la Cultura de paz en las personas escolarizadas, por medio de la Cátedra de Paz; se la considera una decisión estratégica, pues, como lo menciona María Zambrano:

La educación constituye un campo privilegiado y lo es porque si en él no se deja crecer esta nueva planta de la paz como estado permanente y «natural» del mundo humano, ésta no podrá darse en verdad. Sin educación para la paz no habrá paz. Si los dirigentes pueden hacerla, sólo la educación puede establecerla. ${ }^{11}$

Esta metáfora sobre la nueva planta de la paz resulta acertada para explicar la confluencia entre educación y paz en Colombia; dado que el segundo concepto debe establecerse en la mente del ser humano como si fuera una semilla en un cultivo, en el cual el maestro, como labrador, a través de la educación, siembra la Cultura de la Paz en la tierra más fértil para lograr un buen fruto, y el terreno más propicio son las subjetividades de los estudiantes. Resulta es-

8 Xesús Jares, Educación para la Paz Su teoría y su práctica (Madrid: Editorial Popular, 1999).

9 David Hicks, Educación para la paz: cuestiones, principios y prácticas en el aula (Madrid: Morata, 1993).

10 Vinceç Fisas Armengol, “Una Cultura de paz”, Bitácora. No. 7 (1999).

11 María Zambrano, “Educación para la Paz", Revista de Educación, No. 309 (1996): 154. 
tratégico, entonces, implantar la necesidad de procurar una sana convivencia, al reconocer que los conflictos forman parte de la naturaleza humana y que los actos de creatividad e imaginación moral pueden llevar al hombre a alcanzar la estabilidad que necesita. ${ }^{12}$ Entonces, sería

Un esfuerzo capaz de consolidar una nueva manera de ver, entender y vivir el mundo, empezando por el propio ser y continuando con los demás, horizontalmente, formando red, dando confianza, seguridad y autoridad a las personas y a las sociedades, ayudando a movilizarlas y a superar sus diferencias. ${ }^{13}$

En ese esfuerzo, el Sistema educativo actual debe modificarse en todos sus niveles para que dialogara con la estructura orgánica y los valores de la paz. En este sentido, se han de desestructurar paradigmas desde la creación de nuevos modelos pedagógicos integrales y holoárquicos.

Esto dado que, en Colombia, se han concebido los modelos pedagógicos segregados, lo cual es una de las razones para que no se consolidara satisfactoriamente una cultura de paz, pues pocos maestros reconocen la oportunidad de concebir un modelo a la medida, que fusionase diferentes estrategias para responder a las necesidades de un entorno diverso, desde un modelo integral. ${ }^{14}$

En general, los modelos pedagógicos los desarrollan o diseñan los maestros, quienes responden a las reflexiones educativas acerca de las relaciones entre las partes del proceso de enseñanza-aprendizaje y la concepción que se tiene de la vida en sociedad; ${ }^{15}$ esto lo hacen basados en sus concepciones, creencias y filosofía de vida, a partir de los aprendizajes adquiridos en su práctica profesional y la investigación.

Entonces, para cambiar el Sistema educativo, en búsqueda de la consolidación de una cultura de paz, la primera condición de posibilidad la deben ofrecer los programas de formación de maestros, que deben enfocarse en formar

12 John Paul Lederach, La imaginación moral: El arte y alma de construir la paz. (Bogotá: Grupo Editorial Norma, 2008).

13 Vicenç Fisas, Cultura de paz y gestión de conflictos (Barcelona: Icaria, 1998), 33.

14 María del Carmen Gabriela Flores Talavera. "Cuatro formas de entender la educación: Modelos Pedagógicos, conceptualización, ordenamiento y construcción teórica”. Educación y Humanismo. Vol. 21. No. 36 (2019): 137-159.

15 Dolly Quinayas Catuche et al. "Modelo pedagógico". Trabajo de grado de Maestría en Educación, Universidad Pontifica Bolivariana, Puerto Caicedo, Putumayo, 2017. 
profesionales que diseñasen modelos para responder al tiempo y al espacio; que resuelvan problemas educativos para mantener y fortalecer la identidad cultural. ${ }^{16}$

Con esta formación, los maestros no concebirán los modelos pedagógicos como camisa de fuerza, sino como ventana de oportunidad que puede abrirse para crear nuevos modelos, más dialógicos, que procurasen una mejor vida en sociedad y que respondieran a las necesidades de su entorno, al poder de la riqueza étnica y cultural y a la diversidad para fomentar el respeto, la equidad y la preservación de las buenas costumbres. ${ }^{17}$

Entonces, el reto consiste en que, en la formación de maestros, la enseñanza de los modelos pedagógicos no se diera como aspectos sin conexión, sino como la oportunidad para hacerlos co-existir en una misma aula de clases, al generar nuevos patrones, que fuesen holísticos e integrales con diferentes enfoques y basados en el diálogo, el cooperativismo y la comprensión, puesto que el quehacer educativo presupone una concepción del hombre y de la sociedad, que exige comprenderlos en su multidimensionalidad y en su integridad. ${ }^{18}$

De este modo, los nuevos modelos modificarán paradigmas, como los de la cultura de violencia, que permean el país y, como resultado, los maestros formarán ciudadanos solidarios, cuidadores de su ambiente y generadores de paz, dado que "surge el modelo pedagógico denominado educación integral, que justamente incluye e integra de manera holística visiones y acciones de diferentes propuestas educativas $[\ldots]$ para educar integralmente al ser humano". ${ }^{19}$

Al tiempo que se incluye al maestro, su formación y desempeño profesional, para la construcción de paz desde la creación de redes o telarañas que generen efectos de multiplicidad, se instauran en la sociedad nuevos valores y propuestas alternativas diferentes a la violencia.

\footnotetext{
16 Teófilo Vento Ramos et al. "Diseño del modelo pedagógico propio ajustado al pensamiento indígena Ticuna” Trabajo de grado de Maestría en Educación. Leticia: Universidad Pontificia Bolivariana, 2018.

17 Quinayas et al., "Modelo pedagógico", 27.

18 Julián de Zubiría-Samper, Los Modelos Pedagógicos (Bogotá: FAMDI, 1994).

19 Flores Talavera, "Cuatro Formas de...", 144.
} 
De este modo, los maestros han de estar preparados para formar en sus estudiantes las condiciones necesarias que les permitieran participar en ese proceso de consolidación de paz y ser ellos el perfil ejemplar de esos niños y jóvenes que se pretenden formar con la Educación para la Paz.

Así, lo más coherente sería pensar que los maestros deben vivenciar los valores y criterios de la paz y desempeñarse, en ese nuevo Sistema educativo, como maestros mediadores, que identifiquen nodos de relacionamiento al propiciar espacios para que las subjetividades confluyeran y generasen propuestas creativas, desde la aceptación de la diferencia, el contraflujo y la oposición.

\section{La Pedagogía de la Memoria: origen y conceptualización}

Para que el maestro pueda generar esa conexión de su conocimiento con los estudiantes, para consolidar una cultura de la paz, puede basarse en la Pedagogía de la Memoria, que es una reflexión ética sobre la memoria como experiencia viva del tiempo; una pedagogía del mundo centrada en la atención, la escucha, la mirada; una transmisión de la durabilidad del mundo a través de imágenes, historias, relatos, ideas y creencias. ${ }^{20}$

En este orden de ideas, el carácter de esta pedagogía radica en que el sujeto ejercite la memoria en colectivo, para reconocer en el pasado factores que se relacionen con lo que sucede en el presente e intentar establecer un nuevo comienzo. Por tanto, la mejor Pedagogía de la Memoria es aquella que avisa, que da pistas y, para hacerlo, mantiene un estrecho vínculo con el desarrollo de la imaginación sensible.

Esta propuesta pedagógica la adaptó, en el contexto suramericano, Graciela Rubio, quien toma el modelo como propuesta para transformar la sociedad chilena, después de la dictadura. Según Rubio, esta pedagogía otorga palabras a los silenciados y busca traer al presente el pasado olvidado; además, es un proceso que rompe la linealidad del hecho histórico, para abrir el relato a las fisuras del devenir local y propio. Igualmente, revierte los espacios de lo público desde la narración, el reconocimiento de las contingencias y de las aspiraciones, y la

20 Bárcena Orbe, "Pedagogía de la Memoria y transmisión del mundo. Notas para una reflexión". 
otredad. Finalmente, abre el presente cerrado en un solo discurso a otras posibilidades, mundos que provienen tanto desde los pasados y de los futuros que reconocen la tensión entre lo dado y lo deseado y buscan nuevas tramas de posibilidad como acción creativa. ${ }^{21}$

Según Rubio, ${ }^{22}$ esta forma de pedagogía es un proceso de diálogo crítico entre memoria e historia, que debe basarse en la dignidad y el respeto por los derechos, en la búsqueda por la justicia, la desnaturalización de la violencia y la vinculación de la ciudadanía con los procesos históricos, por medio de la narración. Así, la Pedagogía de la Memoria promueve la comprensión y atribución de sentido alojados en el sujeto, en su capacidad de elaborar un juicio político, como un modo de asumir los pasados de dolor y romper el silencio.

Por su parte, Susana Sacavino ${ }^{23}$ presenta esta pedagogía como un proceso que permite a los sujetos adquirir vínculos significativos con el pasado e imaginar y construir futuros más justos, desde el proceso de recordar con los otros, como situar a los sujetos en una relación dialógica que, por medio de la experiencia, permitiera unir ética, política y conocimiento con una mirada crítica, por medio de cuatro ejes pedagógicos: 1) vínculo pasado-presente, 2) desarrollo de una memoria crítica, 3) construcción de un pensamiento reflexivo y crítico y 4) promoción de una cultura de los derechos humanos.

El proceso de implementación de la Pedagogía de la Memoria, en Colombia, se puede evidenciar en diferentes desarrollos investigativos, que lo han utilizado como base. ${ }^{24}$ Estos trabajos han interpelado el quehacer ético-político de

${ }^{21}$ Graciela Rubio, "Educación y memoria. Desafíos y tensiones de una propuesta", Nómadas. Critical Journal of Social and Juridical Sciences. Vol. 15. No. 1 (2007): 175.

22 Graciela Rubio, "Memoria hegemónica y memoria social. Tensiones y desafíos pedagógicos en torno al pasado reciente en Chile", Revista colombiana de educación. No. 71 (2016): 109135.

23 Susana Sacavino, "Pedagogía de la Memoria y educación para el "nunca más" para la construcción de la democracia”, Folios, Segunda época. No. 41 (2015): 69-85.

24 Las investigaciones a las que se refiere el texto son: Vélez Villafañe (2012), Herrera (2013), Ortega, Merchán y Vélez (2014), Vélez Villafañe y Herrera (2014), Londoño Sánchez y Carvajal Guzmán (2016), Murillo Arango (2015), Cantor (2016), Flórez Herrera (2016), Mayorga, López, Romero, Muñoz, Aranzazu (2017); Vásquez-Cangas (2017), Mejía (2017), Caballero (2017), Mora Cepeda (2018), Pérez (2018), Flórez Orellano y Valencia Ibarra (2018), Cárdenas Alvarado y Cruz Bate (2018), Cubillos Vega y Llanán Nogueira (2018) y Romero (2018). 
la escuela en la tramitación pedagógica del pasado y reafirman la relación entre pedagogía y memoria desde la alteridad, el reconocimiento activo de las víctimas y sus derechos y el reto de hacer partícipe a la sociedad en sus reivindicaciones.

Igualmente, evidencian cómo crear experiencias sensitivas, vivenciales y puentes empáticos y se convierte en una estrategia que facilita la compasión y la convivencia, desde un aprendizaje significativo, que contribuye a la construcción de Cultura de paz y a la formación de Competencias Ciudadanas.

Asimismo, estas investigaciones ponen la oralidad y la narración en una posición privilegiada como herramientas didácticas que suscitan la imaginación, dado que la Pedagogía de la Memoria busca ante todo provocar una actitud de descubrimiento de la palabra negada por el silencio que impone la guerra, bajo el método de la mediación.

Adicionalmente, estos trabajos investigativos evidencian el compromiso ético-político y estético de este tipo de pedagogía, lo que conlleva la comprensión del dolor del otro, para llevarlo a niveles de empatía que posibilitan la imaginación y la recreación en la mente de quienes se acercan a ese pasado. ${ }^{25}$

Es una pedagogía que establece una relación entre memoria e historia, que reflexiona sobre la importancia de los derechos humanos y la construcción de la escuela como un territorio de paz y transformación, ${ }^{26}$ desde la idea del acontecimiento que, a partir de ciertos ejercicios en el aula de clase, problematiza el propio mundo, convierte el encuentro en un acontecimiento y genera situaciones de crisis en los estudiantes, por medio de las cuales logran problematizar su vida cotidiana y su relación con el conflicto.

En suma, la Educación para la Paz, mediatizada en la Pedagogía de la Memoria, debe tener como fin la emancipación de las personas, al promover actitudes positivas en torno a sus valores y fomentar la reflexión crítica. Para esto,

$25 \quad$ Noemí Pérez Martínez, "De la memoria a las memorias: Una reflexión teórico-metodológica en torno a la relación entre memoria y educación en derechos humanos", Miradas. Vol. 1. N ${ }^{\circ}$ 1 (2018): 96-115.

26 Luis Felipe Caballero Dávila, "Hacia una Pedagogía de la Memoria, el desacuerdo y el acontecimiento: El caso de la galería de la memoria "somos protagonistas de la historia" del Colegio IED Tibabuyes Universal”, Cambios y Permanencias. Vol. 8. No. 2 (2017): 1109-1124. 
ha de considerar aspectos como: 1) partir del contexto y la experiencia, 2) formar comunidades que recuerden, 3) generar memorias alternativas, todas ellas desde la formación de subjetividades ético-políticas comprometidas y responsabilizadas con el otro. ${ }^{27}$

\section{EI maestro como mediador para la construcción de paz}

En palabras de Jesús Martín-Barbero, ${ }^{28}$ la mediación es la coexistencia entre ruptura y puente, brecha que se cierra debido al lenguaje como objeto que media en la comunicación. El lenguaje está constituido por signos y símbolos que ponen en diálogo a todos los sujetos; de este modo, la comunicación se desarrolla desde la visión de un proceso que logra establecer la cultura de paz en los imaginarios colectivos de los colombianos, a través de puentes lingüísticos de mediación.

El maestro ha de apropiarse de algunos compromisos como mediador en la elaboración de esos puentes lingüísticos, que ponen en común los significados sobre paz, pues los sujetos inmersos en el acto educativo han de reconstruir la convicción y el deseo de la cultura de paz.

En esta articulación, una tarea para el maestro es disminuir la violencia en sus relaciones a través de la construcción de un ámbito, en el cual cada uno pueda expresarse y avanzar en las relaciones, la comunicación y el inter-aprendizaje, ${ }^{29}$ desde el lenguaje, el encuentro y la experiencia. ${ }^{30}$ Otra tarea es asumir el rol de intérprete-mediador, que ponga en común las significaciones sobre los significados de paz que tienen los sujetos interlocutores en el aula. Adicionalmente, ha de problematizar a los estudiantes el contenido que los mediatiza a través de la motivación y las emociones para, por último, generar una toma de conciencia que traiga como resultado final el aprendizaje significativo.

27 Javier Ulises Flórez Herrera, "El uso de la Pedagogía de la Memoria en la construcción de subjetividades desde la perspectiva ética-polítca, un caso en la educación de las élites". Tesis de Maestría en Educación, Universidad Pedagógica Nacional, 2016.

28 Jesús Martín-Barbero, De los medios a las mediaciones: Comunicación, cultura y hegemonía, (Barcelona: Gustavo Gili, 1987); Jesús Martín-Barbero, La educación desde la comunicación (Bogotá: Norma, 2003); Jesús Martín-Barbero, "Retos culturales: de la comunicación a la educación", Nueva Sociedad. No. 169 (2000): 33-43.

29 Prieto, "La comunicación en la educación"

30 Larrosa, "Entre las lenguas: lenguaje y educación después de Babel" 
En definitiva, el rol que el maestro ha de desempeñar es de mediador, interlocutor, intérprete y traductor entre los sujetos que conforman esta sociedad, para así cerrar las brechas existentes, que aumentan la violencia y no permiten la construcción de Cultura de paz. El maestro debe ser el sujeto que conjugue la Educación para la Paz con la Comunicación desde la Pedagogía de la Memoria, para dejar ancladas en la historia: ideologías, creencias y saberes que propendan por una paz sostenible. Adicionalmente, debe ser quien favorezca nuevas conductas y pensamientos, para que los sujetos sean capaces de relacionar pasado, presente y futuro.

En este sentido, Oscar Saldarriaga ${ }^{31}$ propone unos compromisos para los maestros en relación con la formación de verdades y sujetos: 1) empoderarse como sujeto de su saber pedagógico, 2) respetar la subjetividad individual y comunitaria de la comunidad educativa que lo alberga, y 3 ) educar desde su subjetividad. Desde esa perspectiva, es un maestro que, desde su ética, le da sentido al contenido y a la forma del acto educativo. Por tanto, desde su perfil, se caracteriza por demostrar que tiene excelente comunicación intra e interpersonal y se aboca a dialogar constantemente con la comunidad educativa.

Se sugiere la mediación ${ }^{32}$ a modo de una postura que el maestro ha de incorporar al utilizar en forma estratégica la comunicación, lo cual lo convierte en ese mediador que interviene para conducir un proceso de consenso. En definitiva, es un maestro comprometido con incluir la diversidad y la alteridad en sus prácticas, para ponerlas en el centro del acto educativo. El maestro es el mediador que, desde lo comunicacional, pone en diálogo subjetividades, culturas y realidades, para la construcción de un conocimiento y la formación de sujetos aptos para la convivencia en un mundo de diferencias.

Ahora bien, ese maestro debe formarse desde una condición ético-política orientada a interrumpir la permanencia de las ecologías violentas. Este tipo de formación lleva a que el acto educativo fuese un proceso lleno de sentido, que garantiza a sus estudiantes un aprendizaje significativo y el desarrollo de un país

\footnotetext{
$31 \quad$ Oscar Saldarriaga Vélez, "Del oficio del maestro. ¿De intelectual subordinado a experto subordinador?" Educación y ciudad. No. 11 (2006): 53-70.

32 Fisas, "Cultura de paz y gestión de conflictos", 33.
} 
más equitativo por la vivencia cotidiana de la cultura de paz. ${ }^{33}$ Este proceso de formación se fundamenta en las necesidades de: 1) abrir la sensibilidad y la reflexión sobre el dolor, el daño y la pérdida en la vida social; 2) concebir el pasado reciente como fondo de experiencia de la existencia social, pasada y presente e 3) incorporar la enseñanza de los Derechos Humanos desde una perspectiva histórica.

Los principios ético-políticos de esta formación son: respeto, solidaridad, compromiso, participación, construcción de futuro y de reconocimiento con el otro en la sociedad, necesarios para generar inclusión y respeto, desde la reconstrucción de la memoria histórica, orientada en la transformación y en la construcción de justicia y dignidad. Para esto, se han de brindar componentes como: 1) partir del contexto y la experiencia, 2) formar comunidades que recuerden y 3 ) generar memorias alternativas, todas ellas desde la orientación de subjetividades ético-políticas, comprometidas y responsabilizadas con el otro.

Desde esos principios y componentes, se sugieren los temas macro, meso y micro de la propuesta de formación para un maestro mediador que consolide la cultura de la paz, pues "pensar la formación de maestros para el presente puede tener un contenido distinto al que proponen las reformas educativas, producidas en un escenario donde las necesidades sociales y los compromisos éticos no se constituyen en una prioridad". 34

33 Graciela Rubio, "El pasado reciente en la experiencia chilena. Bases para una Pedagogía de la Memoria". Estudios Pedagógicos. Vol. 38. No. 2 (2012): 375-396.

34 Sandra Patricia Rodríguez Ávila, "Formación de maestros para el presente: memoria y enseñanza de la historia reciente”. Revista colombiana de educación. No. 62 (2012): 183. 
Tabla 1. Temas de formación para maestros mediadores.

\begin{tabular}{|l|l|l|}
\hline Temas Macro & \multicolumn{1}{|c|}{ Temas Meso } & \multicolumn{1}{c|}{ Temas Micro } \\
\hline Cultura de paz & $\begin{array}{l}\text { Cultura } \\
\text { Memoria colectiva } \\
\text { y Memoria histórica }\end{array}$ & $\begin{array}{l}\text { - Resolución pacífica de conflictos } \\
\text {-Proyecto de vida y prevención de riesgos } \\
\text { - Proyectos de impacto social } \\
\text { - Hacer memoria en Colombia } \\
\text { - Identidad cultural } \\
\text { - El rol de las mujeres y el asunto de género } \\
\text { - Las estrategias de resistencia }\end{array}$ \\
\hline $\begin{array}{l}\text { Educación para la } \\
\text { Paz }\end{array}$ & $\begin{array}{l}\text { Contexto } \\
\text { socioeconómico } \\
\text { Desarrollo Sostenible }\end{array}$ & $\begin{array}{l}\text { - Historia del conflicto armado colombiano y } \\
\text { de los acuerdos de paz } \\
\text { - Pedagogía de la Memoria } \\
\text { - Dilemas morales } \\
\text { - Uso sostenible de los recursos tanto cultura- } \\
\text { les como naturales } \\
\text { - Prevención del acoso escolar }\end{array}$ \\
\hline Derechos Humanos & Territorio & $\begin{array}{l}\text { - Justicia y Derechos Humanos } \\
\text { - Participación política } \\
\text { - Diversidad y pluralidad } \\
\text { - Impactos y daños del conflicto armado co- } \\
\text { lombiano }\end{array}$ \\
\hline
\end{tabular}

Fuente: esta investigación

Si se reconoce que todo acto educativo es per se un acto comunicativo, entonces la construcción de la paz, desde el Sistema educativo colombiano, requiere de maestros que no tengan como único objeto de reflexión sobre su práctica la pedagogía, sino una comunicación que, por sí misma, tendría que ser pedagógica.

Así, este proceso de consolidación de cultura de paz requiere a un maestro que logre situarse en una relación de saber crítico y ético frente a los acontecimientos históricos y a la manera en que se narran, explican, seleccionan, reacomodan $\mathrm{u}$ omiten.

El maestro-mediador tiene, pues, un rol protagónico en esa consolidación como facilitador de los procesos pedagógicos y, en tal sentido, se lo llama a abrir espacios de reflexión conjunta en los contextos escolares para movilizar el cambio cultural, a la par que debe liderar este cambio, dados su potencial humano y su formación. Este es un compromiso institucional y requiere el interés de todas las instancias para contribuir a este cambio cultural. 
En consecuencia, el ejercicio del rol protagónico de los maestros-mediadores, en este contexto, depende del nivel de compromiso que se adquiera y se visibilizará al constituirse en sujetos democráticos y pacíficos, dispuestos a pasar de modelos autoritarios y rígidos a dialógicos, con prácticas como escucha activa, comunicación asertiva y afectiva y respeto por la diferencia. Esto implica un tipo de formación diferente a la disciplinar y académica que reciben los docentes, puesto que ellos tienen interiorizada la cultura patriarcal violenta, puesto que los educan bajo estos preceptos. ${ }^{35}$

Por tanto, la formación de los maestros debe propender porque ellos, más allá de moldear y modelar conductas, se apasionasen por la reflexión crítica. Más allá de ser unos simples facilitadores, fuesen unos mediadores entre el estudiante y el conocimiento, ${ }^{36}$ al crear ambientes estimulantes para vivir una cultura de paz $\mathrm{y}$, a la vez, fuesen unos profesionales autónomos, que investigan sobre sus propias prácticas en pro de esta necesidad social.

Finalmente, se deben formar maestros que comprendieran que tanto ellos como los estudiantes aprenden desde la experiencia y las emociones, que la escuela se convierte en un espacio colaborativo y que ella misma trasciende sus muros; unos maestros que vivan el diálogo como medio que establece una relación horizontal basada en el amor, la humildad, la esperanza y la confianza, para que reconozcan al estudiante en su rol de conductor activo de sus propios aprendizajes. $^{37}$

\section{Conclusiones}

Este proceso de investigación presenta las articulaciones posibles entre Educación para la Paz (EpP), Pedagogía de la Memoria y Comunicación, como elementos para una propuesta de formación del maestro-mediador para la construcción de paz: La Educación y la Comunicación facilitan el encuentro de individualidades para la formación de subjetividades en el aula, las que, desde la

\footnotetext{
35 Paola Ayala Medina et al., "Despliegue de capacidades humanas de los docentes para construir cultura de paz en la escuela línea de justicia y convivencia escolar Isis". Tesis de Maestría en Educación, Pontificia Universidad Javeriana, 2016. 117-118.

36 Martha Patricia Vives Hurtado, "Modelos pedagógicos y reflexiones para las Pedagogías del Sur.» Boletín Redipe. Vol. 5. No. 11 (2016): 40-55.

37 Vives-Hurtado, "Modelos pedagógicos y...", 40-55.
} 
reflexión crítica, aportan a la construcción y consolidación de una paz sostenible. Debido a esta articulación, se pueden constituir redes o telarañas de relacionamiento, que fusionasen necesidades e intereses en común entre la comunidad educativa, y entre ella y el contexto que la rodea. Por otro lado, los medios de comunicación se convierten en oportunidades para que el maestro se formase y formara, al efectuar una lectura crítica de la realidad y generar espacios de diálogo que resulten en mediaciones para la paz.

Mediante la comunicación, la Pedagogía de la Memoria puede generar vínculos significativos con el pasado, disponer sujetos en una relación dialógica y establecer una cultura de paz que, desde la experiencia, la emoción, la motivación y la empatía, logre que los sujetos del proceso educativo se identifiquen con los símbolos, signos, oralidades y narrativas de la Educación para la Paz, al recurrir a la memoria como sistema simbólico, escenario para la construcción de imaginarios colectivos que elaboren puentes de intersubjetividad.

Por su lado, la articulación entre EpP y Pedagogía de la Memoria facilita el desarrollo de la autonomía y la confianza de los sujetos en formación, lo que garantiza la no repetición de hechos violentos desde la auto-reflexión crítica. Para esto, es fundamental que los maestros respondan a las necesidades del posacuerdo y se formen en Pedagogía de la Memoria para que reconozcan sus alcances y limitaciones.

La articulación clave entre Educación para la Paz, Pedagogía de la Memoria y Comunicación permitirá que el Sistema educativo elimine al máximo la violencia de su estructura, desde el lenguaje, las relaciones y la experiencia. Para lograrlo, se ubica en el centro a un maestro-mediador que debe formarse como un sujeto con una condición ético-política y estética apta, para forjar, desde la experiencia, aprendizajes significativos que lleven a sus estudiantes a vivenciar y promover la cultura de paz.

Por último, los resultados de esta investigación son pertinentes y relevantes para la comunidad académica y científica, en la medida en que, desde las experiencias de base, se ofrece una propuesta de formación para un maestro-mediador perfilado para la construcción de una sociedad que desea convivir en armonía. Además, ofrece una triada articulada que no se encuentra presente en lo encontrado hasta el momento en la literatura, lo que brinda al maestro nuevas 
herramientas, que le permiten mejorar su comunicación en el accionar diario. Futuros procesos de investigación, en esta línea, pueden ahondar en temas como la empatía que debe tener un maestro en Colombia para consolidar formas no violentas de estar en sociedad, desde el manejo de la inteligencia emocional. 


\section{Referencias}

Bárcena Orbe, Fernando. "Pedagogía de la Memoria y transmisión del mundo. Notas para una reflexión”. Con-ciencia social. No. 15 (2011): 109-118.

Caballero Dávila, Luis Felipe. "Hacia una Pedagogía de la Memoria, el desacuerdo y el acontecimiento: El caso de la galería de la memoria "somos protagonistas de la historia” del Colegio IED Tibabuyes Universal”. Cambios y Permanencias, vol. 8. No. 2 (2017): 1109-1124.

Cantor Parra, Luisa Fernanda. "Formación ético-politica, infancia y contexto escolar: comprensiones y aportes desde la Pedagogía de la Memoria". Tesis de Maestría, Universidad Pedagógica Nacional, 2016.

Cárdenas Alvarado, Carolina, y Cruz Bate, Jasmín Rocío. “Tejiendo redes de paz. La memoria como camino para la construcción de culturas de paz". Cambios y Permanencias, vol. 9. No. 1 (enero-junio 2018): 839-850.

Cubillos Vega, Carla, y Llanán Nogueira, Julio. "Pedagogía de la Memoria y escritura performativa en la educación en Derechos Humanos". Revista Latinoamericana de Derechos Humanos, vol. 29, No. 1 (2018): 29-35.

De Zubiría-Samper, Julián. Los Modelos Pedagógicos. Bogotá: FAMDI, 1994.

Fisas, Vicenç. Cultura de Paz y Gestión de Conflictos. Barcelona: Icaria, 1998.

Fisas, Vicenç. Educar para una cultura de paz. Barcelona: Ediciones Bellaterra Cuadernos de construcción de paz, 2011.

Fisas, Vicenç. "Una Cultura de Paz". Bitácora. No. 7 (1999).

Flores Talavera, María del Carmen Gabriela. "Cuatro formas de entender la educación: Modelos Pedagógicos, conceptualización, ordenamiento y construcción teórica.» Educación y Humanismo, vol. 21. No. 36 (2019): 137159. 
Flórez Herrera, Javier Ulises. "El uso de la Pedagogía de la Memoria en la construcción de subjetividades desde la perspectiva ética-polítca, un caso en la educación de las élites". Trabajo de grado de Maestría en educación, Universidad Pedagógica Nacional, 2016.

Flórez Orellano, Jean Carlos, y Valencia Ibarra, Mónica. "Pedagogía de la Memoria y de la paz: herramientas para la implementación de la memoria histórica y la oralidad en la enseñanza de la historia escolar". Cambios y Permanencias, vol. 9. No. 1 (2018): 898-912.

Herrera, Martha Cecilia. "Trazos sobre una Pedagogía de la Memoria”. En: Memoria y formación: configuraciones de la subjetividad en ecologías violentas, Herrera, Martha Cecilia. Bogotá: Universidad Pedagógica Nacional, 2013. 175-198.

Hicks, David. Educación para la paz: cuestiones, principios y prácticas en el aula. Madrid: Morata, 1993.

Jares, Xesús R. Educación para la Paz. Su teoría y su práctica. Madrid: Editorial Popular, 1999.

Larrosa, Jorge. "Experiencia y Pasión”. En: Entre las lenguas: lenguaje y educación después de Babel, Jorge Larrosa. Barcelona: Laertes, 2003. 165-178.

Lederach, John Paul. La imaginación moral: El arte y alma de construir la paz. Bogotá: Norma, 2008.

Londoño Sánchez, Juan Guillermo, y Carvajal Guzmán, Jeimmi Paola. "Pedagogías para la memoria histórica: reflexiones y consideraciones para un proceso de innovación en el aula". Educación y ciudad. No. 30 (Enero - Junio 2016): 65-78.

Martín-Barbero, Jesús. De los medios a las mediaciones: Comunicación, cultura y hegemonía. Barcelona: Gustavo Gili, 1987.

Martín-Barbero, Jesús. La educación desde la comunicación. Bogotá: Norma, 2003. 
Martín-Barbero, Jesús. "Retos culturales: de la comunicación a la educación". Nueva Sociedad. No. 169 (2000): 33-43.

Mayor Zaragoza, Federico. "Educación para la paz". Educación XXI. No. 6 (2003): 17-24.

Mejía, Marco Raúl. "Entre el conflicto y la memoria: sistematización de experiencias geo-pedagógicas en Colombia post-acuerdo por la paz". Revista de Educación de Adultos y Procesos Formativos. No. 5 (2017): 63-87.

Mora Cepeda, Lina María. "Pedagogía de la Memoria para abordar la cátedra de la paz”. Cambios y Permanencias, vol. 9. No. 1 (2018): 913-927.

Murillo Arango, Gabriel Jaime. Narrativas de experiencia en educación y Pedagogía de la Memoria. Buenos Aires: Editorial de la Facultad de Filosofía y Letras Universidad de Buenos Aires, 2015.

Ortega Valencia, Piedad et al. "Enseñanza de la historia reciente y Pedagogía de la Memoria: emergencias de un debate necesario". Pedagogía y Saberes. No. 40 (2014): 59-70.

Pérez Martínez, Noemí. "De la memoria a las memorias: Una reflexión teórico-metodológica en torno a la relación entre memoria y educación en derechos humanos". Miradas. Revista de Investigación, vol. 1. No. 1 (2018): 96-115.

Prieto, Daniel. La comunicación en la educación. Buenos Aires: Ediciones Ciccus La Crujía, 1999.

Quinayas Catuche, Dolly et al. "Modelo pedagógico". Tesis de maestría, Universidad Pontificia Bolivariana, 2017.

Rodríguez Ávila, Sandra Patricia. "Formación de maestros para el presente: memoria y enseñanza de la historia reciente". Revista Colombiana de Educación. No. 62 (2012): 165-188. 
Rubio, Graciela. "Educación y memoria. Desafíos y tensiones de una propuesta". Nómadas. Critical Journal of Social and Juridical Sciences, vol. 15. No. 1 (2007): 163-175.

Rubio, Graciela. "El pasado reciente en la experiencia chilena. Bases para una Pedagogía de la Memoria". Estudios Pedagógicos, vol. 38. No. 2 (2012): 375-396.

Rubio, Graciela. "Memoria hegemónica y memoria social. Tensiones y desafíos pedagógicos en torno al pasado reciente en Chile". Revista colombiana de educación. No. 71 (2016): 109-135.

Sacavino, Susana. "Pedagogía de la Memoria y educación para el "nunca más" para la construcción de la democracia". Folios, Segunda época. No. 41 (2015): 69-85.

Saldarriaga, Oscar. "Del oficio del maestro. ¿De intelectual subordinado a experto subordinador?". Educación y Ciudad. No. 11 (2006): 53-70.

Strauss, Anselm, y Juliet Corbin. Bases de la investigación cualitativa. Técnicas y procedimientos para desarrollar la teoría fundamentada. Medellín: Universidad de Antioquia, 2002.

Torres Taborda, Sandra Liliana, y Martínez Meneses, Efraín José. Formación de formadores para el posconflicto: educación mediada por TIC para la paz. Sabaneta: Fondo Editorial Unisabaneta, 2017.

Vázquez-Cangas, Verónica. "De imágenes, sentido y paz. Una esperanza llamada Colombia”. (pensamiento), (palabra)... y obra. No. 17 (Enero-Junio 2017): 6-17.

Vélez Villafañe, Gerardo. "Pedagogías de las memorias de la historia reciente colombiana: ¿construir memoria, en el campo de una memoria imposible?”. Revista colombiana de educación. No. 62 (2012): 245-264.

Vélez Villafañe, Gerardo, y Martha Cecilia Herrera. "Formación política en el tiempo presente: ecologías violentas y Pedagogía de la Memoria". Nómadas. No. 41 (Octubre 2014). 
Vento Ramos, Teófilo et al. "Diseño del modelo pedagógico propio ajustado al pensamiento indígena Ticuna". Tesis de maestría, Universidad Pontificia Bolivariana, 2018.

Vives-Hurtado, Martha Patricia. "Modelos pedagógicos y reflexiones para las Pedagogías del Sur". Boletín Redipe, vol. 5. No. 11 (Octubre 2016): 40-55.

Zambrano, María. "Educación para la Paz". Revista de Educación. No. 309 (1996): 151-159. 